\title{
Analysis of room overpressure after launching an integrated extinguishing system
}

\author{
Lukáš Hurda ${ }^{1, *}$, Richard Matas ${ }^{1}$, and Marek Redmer ${ }^{2}$ \\ ${ }^{1}$ University of West Bohemia in Pilsen, New Technologies - Research Centre, Univerzitní 2732/8, 30100 Pilsen, \\ Czech Republic \\ 2 Safran Cabin CZ s.r.o., Univerzitní 1119/34, 30100 Pilsen, Czech Republic
}

Received: 31 March 2019 / Accepted: 3 November 2019

\begin{abstract}
The throttling of liquefied substances from high pressure vessels to an enclosed volume starting at atmospheric pressure is described in order to determine the thermodynamic state of gaseous contents of the extinguished room. A time dependent, 0D mathematical model is implemented describing the state inside the agent container, the isenthalpic throttling in the distribution system, agent vaporization and mixing with air. The agent is modelled as real gas, and solid-vapour two phase behaviour is approximated. Other influences on the process including the heat transfer from selected solid parts inside the room and the gas mixture leakage out of the room are taken into account. The main outcome is an MS Excel tool for the design optimization of integrated fire extinguishers. The optimization balances two contradictory requirements: volumetric concentration of the agent to sustain the fire extinguishing capabilities, and tolerable room overpressure. Agent fill weight and discharge time are adjusted. The discharge time is controlled by the distribution piping and design of the spray nozzles. System operation is checked with regards to various initial and boundary conditions.
\end{abstract}

Keywords: Fire suppression / gas outflow from vessel / triple point

\section{Introduction}

The design tool for an integrated fire extinguishing system is described. Many aspects of the process are covered by standards but concerns about the emerging overpressure in the extinguished volume require careful analysis and a proposal of new techniques. in [1].

This system and process was already briefly discussed

The thermodynamic system consists of two main parts: an enclosed volume (a room) and extinguishing agent container (a pressure vessel)

The room is initially filled with clean, dry air at atmospheric or near-atmospheric pressure and ordinary temperature. The heat transfer between the walls and the surroundings is omitted completely as the real-life object being modelled is well insulated and relatively low temperature gradients are expected. The model is not completely closed regarding mass conservation. The simplest model of gas mixture leakage based on constant dimensions of a virtual nozzle $i$ is discussed and sophisticated leakage model based on measurement data is implemented.

\footnotetext{
* e-mail: hurda@ntc.zcu.cz
}

The vessel may have a different initial temperature than the room. The heat transfer through the vessel wall is also neglected as the conditions for its evaluation are unclear. The surface-to-volume ratio of pressure vessels of common types is relatively low, so the heat transfer should not have a large influence on the relatively fast process, even though the vessel materials are usually highly conductive and relatively large temperature gradients may occur. The vessel is installed in a separate compartment of the room, which prevents sudden changes in environment temperature. Also, the heat being transferred in the whole process has similar effect and the same magnitude whether it is conducted through the vessel wall, the walls of the distribution system piping or exchanged in direct contact with sprayed droplets.

The room is installed with a piping system ending with nozzle sets for agent distribution. This parts' heat capacity and heat transfer properties are neglected for similar reasons as for the vessel. The whole distribution system is modelled only in terms of pressure drop.

Real gas thermodynamic data for three substances from the NIST REFPROP database are used for various calculations of agent state change: $\mathrm{CO}_{2}$ and two hydrocarbon/ halocarbon mixtures. These mixtures are used for approximating a pure compound used in reality that is 
not available in the properties database. The substitutes were chosen based on their interchangeability in refrigeration applications as stated in [2]. The agent is expected to be liquid initially.

The proper functioning of the fire extinguishing system is ensured by a sufficient volumetric concentration of agent in the extinguished volume, it is checked throughout the time-dependent calculation. The unusual limit of the overpressure comes from the demand for integrity of the lightweight structural design of the room walls. Such walls are also highly pliable and can deflect while introducing room volume expansion by work expelled by gas and enlargement of the slots which mediate the agentair mixture leakage out of the room. The latter effect is discussed and addressed.

\section{Thermodynamic state of extinguishing agent in the pressure vessel}

The main reason for being concerned with the process going on during the emptying of the bottle is to find the starting point of isenthalpic expansion (throttling) which occurs in the piping and mainly in the nozzles (see Sect. 3.1), as this enables the enthalpy of the released agent to be determined.

The vessel is designed to release only the liquid phase of the agent, even though the agent enters the two-phase region just after the start of release. This is ensured by a siphon tube or a bottom release socket. The presence of relatively well separated liquid and vapour phases is expected, despite the ongoing boiling process in the vessel.

The state of the released agent is then found on the liquid saturation curve. How the agent pressure and mass drops with time also needs to be examined, then the state of agent in the bottle can be determined in discrete time levels.

\subsection{Initial release of pressure in fluid}

In the first moment after the container socket is opened, the pressure drops quickly as the contents of the container are mostly incompressible. In reality, it is necessary for the vessel to be filled with a small fraction of inert gas to compensate for the temperature changes of the vessel contents, but this fraction occupies too small volume to have a significant effect on the pressure release.

The first time step of the calculation finds the point on the liquid saturation curve which is reached by isentropic expansion of fluid from the prescribed initial pressure and temperature, see (1). The isentropic nature is expected as the kinetic aspects of the process are minor.

$$
p_{\text {sat }}=f\left(s_{\text {sat }}\right) ; s_{\text {sat }}=s_{\text {init }}=f\left(p_{\text {init }}, T_{\text {init }}\right) .
$$

\subsection{Mass flow estimation}

An important assumption is made that the mass flow rate is constant during most of the agent discharge process. This conforms with $[3,4]$. With this assumption, the value of the

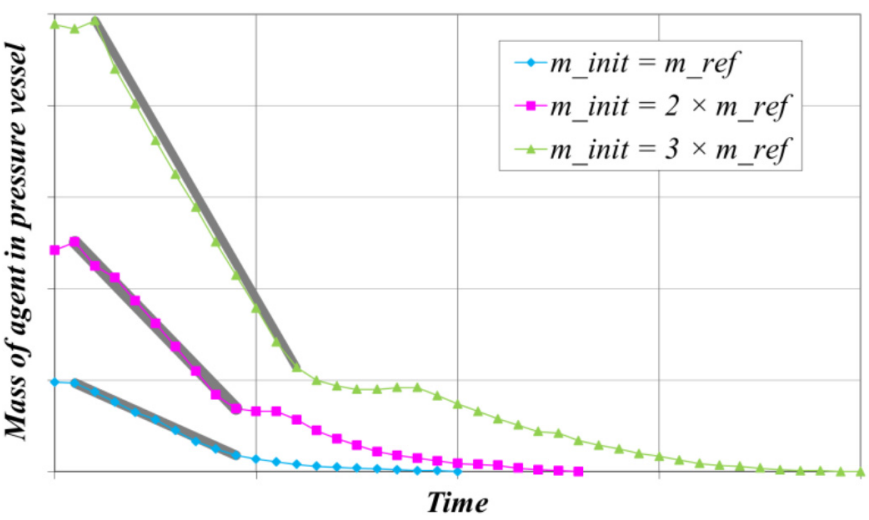

Fig. 1. Discharge process for three experimental tests on a room prototype with $\mathrm{CO}_{2}$ used for verification of the constant mass flow rate. Thick grey lines indicate the linear approximation of mass, i.e. approximation of constant mass flow rate.

mass flow rate can become an input quantity for the design of the distribution system, so it is not calculated in any way, but may be a subject of optimization.

An explanation why the two phase, boiling mix discharge rate does not vary with dropping pressure in the container may lie in the subsonic design of the spray nozzles which control the flow speed and the two-phase state of the agent in front of the nozzles allowing the compressible flow to occur.

The fluid definitely becomes a two-phase mixture before reaching the nozzles. The partial evaporation is caused by a pressure drop in the parts of the distribution system upstream from the nozzle (probably a minor drop compared to nozzle) and the heat transfer through the piping walls delivering certain amount of latent heat to the flowing agent. It is not known what determines the constant mass flow rate and if the expansion in the nozzles is dominated by liquid, gas or complex two-phase behaviour. This would require tedious research. For this purpose, the experimental verification (see Sect. 2.3) and similar experience of other authors is considered satisfactory.

In case of gas dominated outflow, the pressure ratio is high enough to choke the flow through the convergent nozzles for most of the two phase fluid release. The critical pressure ratio ranges between 0.55 and 0.58 for all subjected gases.

The end of the discharge is determined by the point where there is only vapour and no liquid in the vessel. The period of subsonic outflow could possibly start near this point and is neglected due to the low total mass of the outflow.

\subsection{Mass flow experimental verification}

The mass flow estimation was verified experimentally. In Figure 1, a constant mass flow rate may be observed for most of the total mass of the agent discharged. The diminishing outflow on the right side of Figure 1 is the part of discharge after the "end of liquid" point. This 


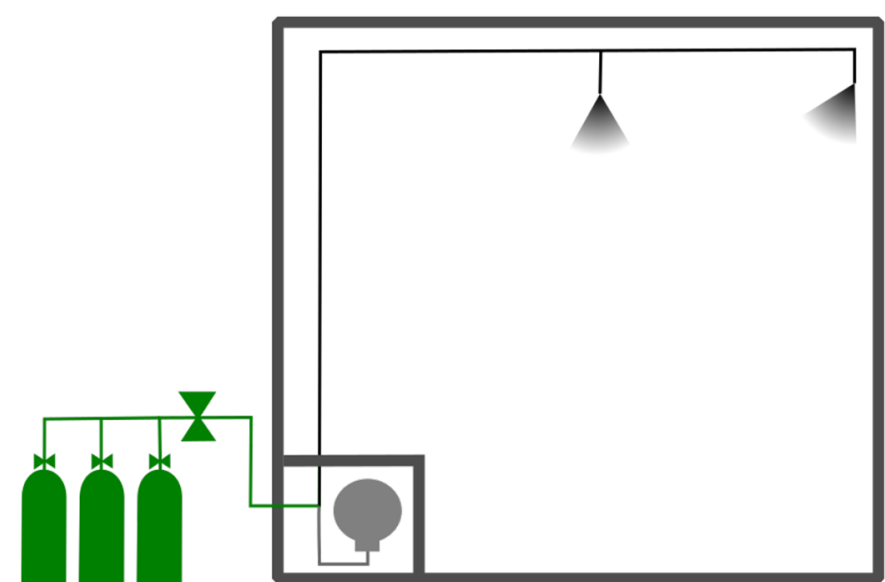

Fig. 2. Simplified experiment layout. Standard integrated pressure vessel (grey) replaced with external pressure bottles of $\mathrm{CO}_{2}$ for parallel discharge controlled initiated by manual release valve (green).

point is approached extremely early in the $\mathrm{CO}_{2}$ test due to reaching the states under the triple point, where the vessel siphon tube freezes fully. This later discharge is neglected in the calculation and the discharge is considered to continue at the prescribed constant flow rate.

The linear parts of the curves from Figure 1 do confirm the mass flow rate is constant, however, for a given distribution system, an equal value of this constant should be observed. This discrepancy lies in the setup of the experiment (see Fig. 2), in which one to three pressure vessels of gas were used. These were standard industrial pressure containers equipped with valves, which are not designed to deplete the content in a matter of seconds. Therefore, the limiting sonic flow occurred first in the parallel-mounted vessel valves. This hypothesis is confirmed by the fact that the mass flow rates for the three cases are approximately in the same ratio as the total initial mass and the number of vessels $1: 2: 3$.

\subsection{Model of state of the agent in the vessel during outflow}

The state change of the agent in the pressure vessel is described with these assumptions: only liquid flows out of the vessel through the bottom outlet and all the vapour is trapped above the liquid level. The second assumption is that the process is adiabatic, which is an estimation since the surface-to-volume ratio of common types of pressure vessels is relatively low and therefore the heat transfer is negligible.

The total volume of agent fill is obtained from the total mass of agent fill and specific volume of the saturated liquid in a state after reaching saturation as described in Section 2.1. The known volume of the container is not considered as a valuable parameter because of the unknown volume of the inert gas fill occupying a portion of the vessel.

\subsection{Isentropic expansion in two-phase region}

The first of the two processes used to describe the liquid release from the vessel mimics the expansion of the remaining contents of the vessel. The concurrent increase of two-phase quality is described in the next subsection.

As the mass flow rate is known, the released mass of the liquid and the related specific volume for each time step is determined. This released mass has the volume of $\Delta m_{e} \cdot v_{\text {eliq }(n-1)}$, which is taken as the total volume change for the analysed isentropic expansion. The specific volume after the assumed (or though) expansion can then be determined as the hypothetically enlarged total volume divided by the current remaining agent mass - as in (2):

$$
v_{e n}=\frac{V+\Delta m_{e} \cdot v_{e l i q(n-1)}}{m_{e n}}
$$

The state after such expansion can then be expressed based on $s_{e n}, v_{e n}$. As the calculation is built in MS Excel, a table-based numerical solution is made. The two-phase quality is quantified for all available points of the saturation curve for both the entropy $s_{e n}$ and the specific volume $v_{e n}$, see $(3)$.

$$
x_{e n s}=\frac{s_{e n}-s_{l i q}}{s_{v a p}-s_{l i q}}, \quad x_{e n v}=\frac{v_{e n}-v_{l i q}}{v_{v a p}-v_{l i q}},
$$

The pressure-temperature level which yields the smallest difference between these two values of quality $\left(x_{e n s}-\right.$ $\left.x_{e n v} \cong 0\right)$ is chosen as a resulting state after fictional isentropic expansion along with the quality $x_{e n s}=x_{e n v}=$ $x_{\text {enpre-release }}$.

The resulting temperature and pressure are those present in the vessel at the end of the time step. The fictional increase of volume compensates for the amount of liquid expelled from the bottle. This decrease of amount of liquid also causes an increase of the two-phase quality which is described below.

\subsection{State change due to liquid release}

As the total volume increase discussed in the previous subsection is only assumed, the mass decrease in the pressure vessel is modelled further. An increase in the twophase quality at constant pressure and temperature is calculated. The siphon tube or bottom socket of the vessel ensures that only liquid is released.

The relation of the quality after assumed expansion (pre-release) to the real quality after the concurrent liquid release and expansion is expressed simply in (4).

$$
x_{\text {en }}=x_{\text {enpre-release }} \cdot \frac{m_{n-1}}{m_{n}} .
$$

This practice is valid if the time step is short enough, so the increase of the quality is small. This may be measured by the difference of the achieved fluid state from the presented calculation and if the expansion is calculated after the liquid release - in the reverse order of that 


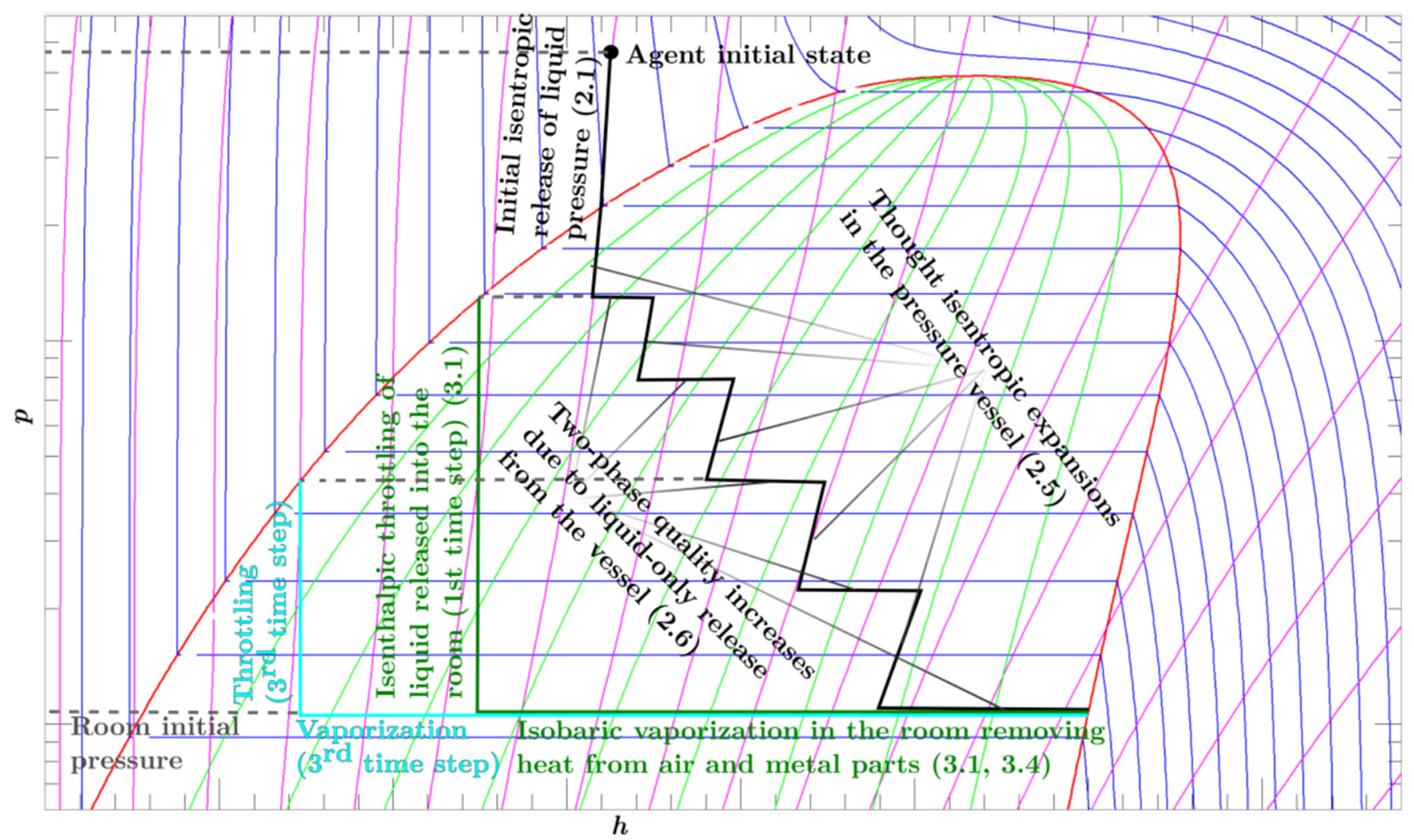

Fig. 3. Schematic pressure-enthalpy diagram of the extinguishing agent with annotated examples of phenomena described in (2) and (3). Red - saturation curves; blue - isotherms; magenta - isentropes, green - curves of constant two-phase quality. Other curves annotated in the figure by text of corresponding colour. (Own work based on NIST REFPROP fluid thermophysical database program.)

presented here. The proposed order was chosen to avoid the unstable behaviour of the quality increase calculation from saturated liquid state, where $x=0$.

\section{Thermodynamic state of gas mixture in the room}

\subsection{Released agent temperature and enthalpy}

The isenthalpic throttling happening in the distribution system always starts at the saturated liquid state determined by the pressure in the vessel. This expansion process ends at the pressure level of the room contents, since the agent vaporizes partially and is therefore in the two-phase region, the discharge temperature is constrained by this pressure. This temperature is lower than the room temperature in most cases.

The remaining latent heat is expected to be transferred to the sprayed agent droplets rapidly from the current gaseous contents of the room. This portion of the specific enthalpy of state change can be evaluated as follows (5) and the obtained value can be illustrated as the length of horizontal part of the green and cyan thick lines in Figure 3:

$$
q_{\text {evap }}=h_{\text {dnvap }}-h_{d n} .
$$

The temperature of the room contents before the mixing calculation is initiated is lowered by the heat consumed by the phase change of the agent as described in equation (6):

$$
T_{n r p r e m i x}=T_{r(n-1)}-\frac{q_{\text {evap }} \cdot \Delta m_{n}}{c_{(n-1) r} \cdot m_{(n-1) r}} .
$$

\subsection{Carbon dioxide under triple point pressure}

There is a serious issue regarding the approach of the two-phase area of $\mathrm{CO}_{2}$ snow - between solid and vapour states. The process of calculation is the same whether the agent phase change is vaporization or desublimation, but as the thermodynamic properties database REFPROP focuses on fluids, the data in the area of solid and the 2-phase solid-vapour area are unavailable. There are only data of the saturated vapour curve as the right-hand side limit of the snow area. Therefore, constant enthalpy and entropy of vaporization is assumed as found in [5].

Under this assumption, REFPROP data on $\mathrm{CO}_{2}$ can be expanded as in Figure 4. Just the values on the saturation curves on both sides of the snow area together with two phase quality value are needed for all necessary evaluations. 


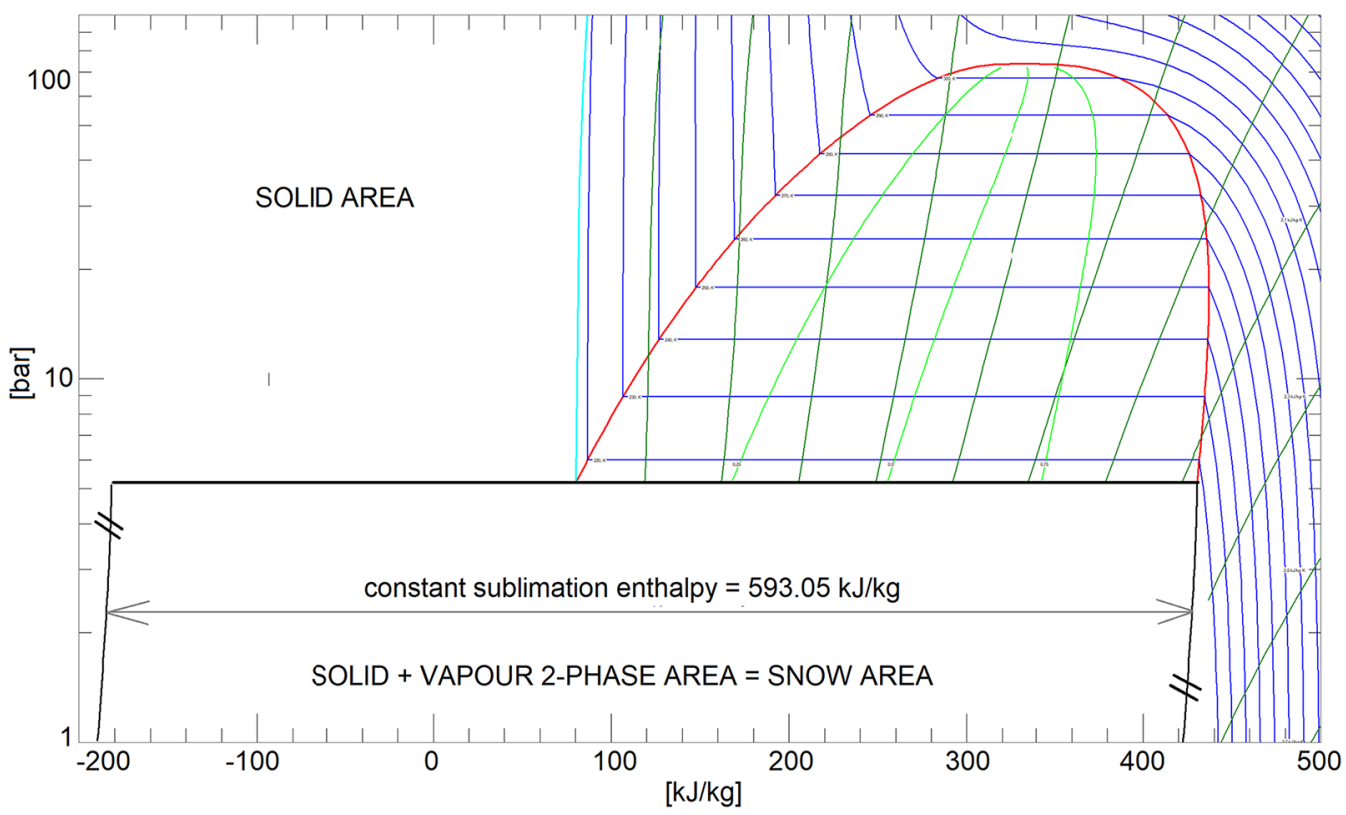

Fig. 4. Illustration of REFPROP data expansion. Cyan: melting line, red: saturated vapour and liquid curves, light green: vapourliquid 2-phase quality isolines, dark green: entropy isolines, blue: isotherms, black: snow area bounds, right curve is part of REFPROP data.

\subsection{Mixing process}

The mixing of the discharged agent with the gaseous contents of the room is estimated using the ideal gas theory. In the first time step, pure air is mixed with the agent and the successive steps are calculated considering the already existing mixture. The agent is considered to be in a saturated vapour state, usually colder than the rest of the room.

The specific heat (7) and specific gas constant (8) of the mixture is a mass weighted average. The temperature is an average weighted by mass and specific heat of the components (9):

$$
\begin{gathered}
c_{p n r m i x}=\frac{c_{p d} \cdot \Delta m_{n}+c_{p n r} \cdot m_{(n-1) r}}{\Delta m_{n}+m_{(n-1) r}}, \\
r_{n r m i x}=\frac{r_{d} \cdot \Delta m_{n}+r_{n r} \cdot m_{(n-1) r}}{\Delta m_{n}+m_{(n-1) r}}, \\
T_{n r m i x}=\frac{T_{d} \cdot c_{p d} \cdot \Delta m_{n}+T_{n r p r e m i x} \cdot c_{p n r} \cdot m_{(n-1) r}}{c_{p d} \cdot \Delta m_{n}+c_{p n r} \cdot m_{(n-1) r}} .
\end{gathered}
$$

$T_{d}$, the agent discharge temperature is already the saturation temperature at room pressure, since the heat exchange during evaporation is considered in the previous step of the calculation described in Section 3.1.

\subsection{Heat addition from metal bodies}

When the room cools down rapidly, some parts may be able to expel its heat content as well as the gases do. There are a few factors to assess the ability to expel heat rapidly.
Bodies for this analysis need to be made of highly conductive materials have thin walls and a large surface area exposed directly to the air. Also, the total amount of heat per Kelvin (mass times specific heat) in such bodies must be high enough to have any impact on the whole process. The eligible parts of a room interior are sheet metal and thin metal profiles.

The temperature distribution in the profile is neglected. A single value of bulk temperature is used. For the initialization of an iterative process, the temperature of the metal part is not expected to change from the previous time step (10).

$$
T_{m n i n i t}=T_{m(n-1)} .
$$

Only two iterations of the process presented in (11), (12) give satisfactory results.

$$
\begin{gathered}
Q_{m n}=\alpha \cdot A_{m} \cdot\left(\frac{T_{m(n-1)}+T_{m n}}{2}-T_{n r}\right), \\
T_{m n}=T_{m(n-1)}-\frac{Q_{m n}}{c_{m} \cdot m_{m}} .
\end{gathered}
$$

A qualified guess must be made for the heat transfer coefficient $\alpha$ estimation. It may lie somewhere between 10 and $50 \mathrm{~W} /\left(\mathrm{m}^{2} \mathrm{~K}\right)$. It can be adjusted concerning the exposure of the metal surfaces to the nozzle wake areas, possible contact with liquid particles of the agent and other factors.

This simple method, without consideration of temperature distribution inside the thin-walls, has been proven to differ less than $1 \%$ from a more complex analytical calculation of transient heat conduction in an infinite 
plate. For the given material parameters of aluminium alloy and a thickness of $1.5 \mathrm{~mm}$, the temperature difference between the exposed surface and opposite adiabatic surface is on the order of $0.01 \mathrm{~K}$.

For an idea of the scale of the results achieved and how notable is the submission of heat from metal parts in the room: $5-10 \%$ of heat supplied to the agent usually comes from metal; the rest is donated by air. After the period of agent discharge, the metal parts are cooled down by $\sim 5 \mathrm{~K}$, whilst mixture-to-metal temperature difference remains above $20 \mathrm{~K}$. Further heat addition from the metal can be expected.

\subsection{Control volume leakage}

The room is not manufactured to be completely airtight. Some slots and chinks may be present in the outer bounds of the room structure. Overpressure acting on the inner side of the room causes the gases to leak. This may have the positive effect of reducing the overpressure. Underpressure may be considered too and it actually occurs during the initial part of fire extinguishing process.

The amount of leak per time step of the calculation is based on the current overpressure, the surroundings are considered to stay at a constant pressure equal to the initial room pressure. Due to the low discharge velocities, incompressible gas dynamics theory is adopted.

The first versions of the model have used an idea of a virtual orifice of constant area, where the flow rate was linearly dependent on the pressure difference. The early pressure test on the room was carried out on a single overpressure level and the results of this test were then used to quantify the virtual orifice area. Since the structure of the room is significantly pliable and the slots may stretch and spread, this approach was abandoned. Instead, sets of pressure tests were completed to obtain curves of flow rate dependency on the variable overpressure.

To illustrate the deformations: the room ceiling deflection measured during room prototype pressure test at cca $30 \mathrm{mbar}$ overpressure reached approximately $50 \mathrm{~mm}$.

For simple implementation, the leakage curves were approximated by second order polynomial. This respects the expectations of gaining quadratic rise of slot area with the linear deflection of the room wall panels.

Two sets of tests were worked. The first one with a carefully manufactured room prototype without any structural changes, sealed as well as possible by the standard manufacturing procedure. This room has experienced a nominal value of leak rate at 1.5 mbar overpressure equal to $3.7 \mathrm{~L} / \mathrm{s}$. The second one was the same prototype with artificially increased leak rate of $8.4 \mathrm{~L} / \mathrm{s}$ at the same overpressure level.

Eight overpressure levels ranging from 1 to $30 \mathrm{mbar}$ were then tested for both nominal leak rate cases and the obtained leak rates were used for polynomial regression. Only the $3.7 \mathrm{~L} / \mathrm{s}$ nominal leak rate case is later used in the calculations, as it is on the safe side considering the higher overpressure is generated more easily with low leak rate values. Secondly, the drilled metal plate, which was used in the case of $8.4 \mathrm{~L} / \mathrm{s}$ nominal leak rate, may not be an accurate representation of a less tight room, especially considering the dynamic behaviour of the leaks.

A leakage curve polynomial derived from $3.7 \mathrm{~L} / \mathrm{s}$ measurement is written in (13). The non-realistic zeroth order coefficient was replaced with zero in calculations. Then the expression does not generate a finite leakage rate at no pressure difference between the room and the environment. The downward shift of the curve, which is applied by this substitution is again an adjustment on the safe side. The overpressure is given in mbar gauge pressure. The values which are generated by this expression are shown in Figures 5 and 6.

$$
\dot{V}_{l}=f\left(p_{r}\right)=0.19 \cdot p_{r}^{2}+0.38 \cdot p_{r}+3.21 .
$$

Underpressure conditions were not tested, so the constant area orifice model was kept for the underpressure cases, where it should be on the safe side of generating intake flow rates possibly above realistic values. The typical slots between flat structural panels should become smaller in this case.

\subsection{Overpressure and volumetric concentration}

Perfect gas behaviour is considered for final overpressure evaluation. The value of $p_{r}$ is adjusted considering the leakage. No iterative process is implemented to correct the influence of overpressure used for leakage quantification. Equation (14) describes the overpressure as a result of isothermal expansion of the gas mixture due to total mass drop by leaks. Section 3.3 clarifies how the specific gas constant and mixture temperature is obtained. The room volume is an input parameter.

An adiabatic model of expansion is possible to employ and may be more accurate. The room temperature $T_{r}$ would then need to be adjusted. Both the non-iterative nature of the calculation and isothermal expansion model call for a short time step to keep the accuracy.

$$
p_{r}=\frac{m_{r} r_{r} T_{r}}{V_{r}} .
$$

The estimation of the volumetric concentration uses the specific volume of saturated vapour of the agent. Only entropy tables were exported to Excel from REFPROP for the vapour single phase area. It is unnecessary to involve other two-parameter tables in the calculation tool just for the concentration evaluation.

\section{Results}

Below the examples of results obtained from the above described calculations are shown. The input parameters are presented in Table 1. Two cases are presented, while the room parameters including the initial state of air, metal parts properties and leakage description remain the same. Two different extinguishing agents are used: $\mathrm{CO}_{2}$ and R403B with different initial weight and pressure, but with the mass flow rate set to achieve $30 \mathrm{~s}$ release time for both cases.

The results are included in Figures 5 and 6 . 


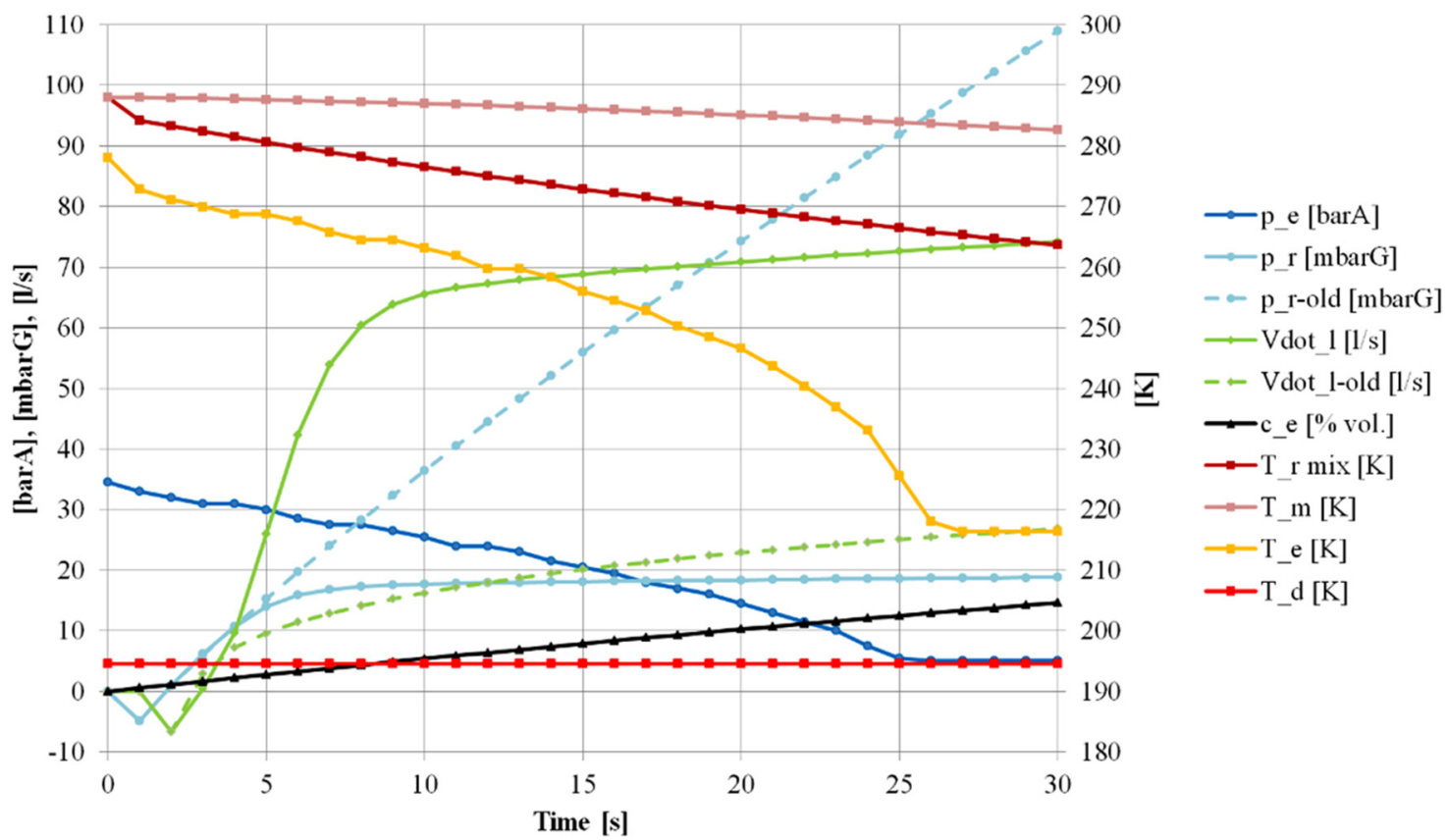

Fig. 5. Results of the $\mathrm{CO}_{2}$ case.

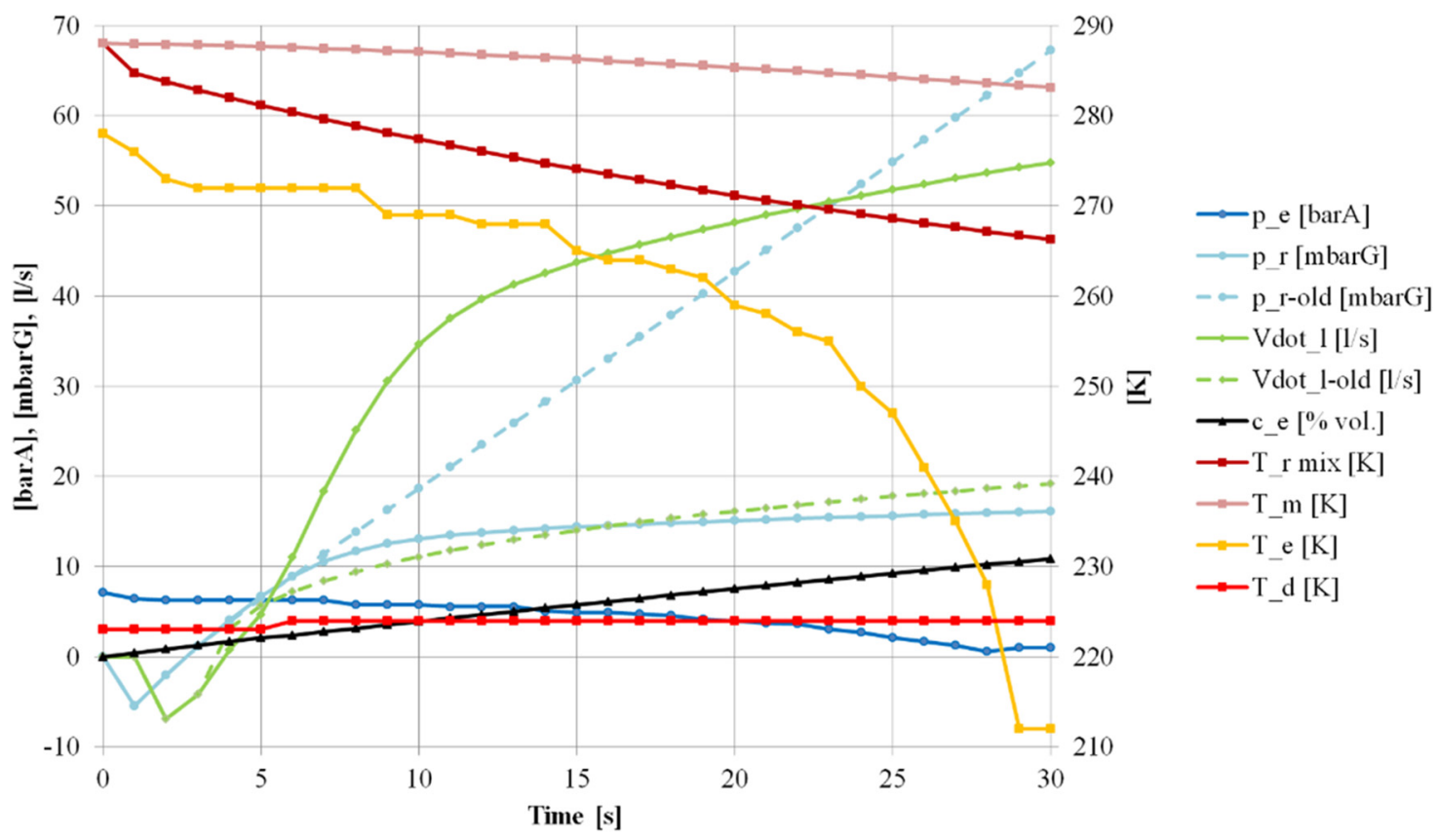

Fig. 6. Results of the R403B case.

The agent pressures $\left(p_{e}\right)$ drop almost linearly along with constant flow rate. The initial pressure release is not visible in the graph and curves for both agents start at the saturation pressure for given state. For $\mathrm{CO}_{2}$, the pressure does not reach under 5,1 barA where lays the triple point. The sudden change of phase change enthalpy on the entry to the snow two-phase area does not have a significant impact on the other processes.
Room overpressure $\left(p_{r}\right)$ and volumetric flow rate of leaks $\left(\dot{V}_{l}\right)$ tell that the rapid cooling of the room content has larger effect to the pressure in the room than the addition of agent mass in the initial period of extinguishing. During this period, the pressure is lowered in the room causing outside air to be taken inside the room. The temporal width of this phase is varying based on the agent type used. 
Table 1. Input parameters of example calculations.

\begin{tabular}{llll}
\hline Type of substance & & $\mathrm{R} 403 \mathrm{~B}$ & $\mathrm{CO}_{2}$ \\
\hline Extinguishing agent & $m_{0}$ e $[\mathrm{kg}]$ & \\
Mass & $T_{0}$ e $[\mathrm{K}]$ & 10 & 278 \\
Initial temperature & $p_{0}$ e $[\mathrm{barA}]$ & 278 & 100 \\
Initial pressure & $\dot{m}_{e}[\mathrm{~kg} / \mathrm{s}]$ & 40 \\
Mass flow rate & $\tau[\mathrm{s}]$ & 0.333 & 30 \\
Release time & $r_{e}[\mathrm{~J} / \mathrm{kg} / \mathrm{K}]$ & 30 \\
Specific gas constant & & 80.52 \\
Air in the room & $p_{0}[\mathrm{bar}]$ & \\
Initial pressure & $T_{0}[\mathrm{~K}]$ & 1.013 \\
Initial temperature & $V_{r}\left[\mathrm{~m}^{3}\right]$ & 288 \\
Room volume & $c_{p r}[\mathrm{~J} / \mathrm{kg} / \mathrm{K}]$ & 12.6 \\
Specific heat & $r_{r}[\mathrm{~J} / \mathrm{kg} / \mathrm{K}]$ & 1005 \\
Specific gas constant & $\rho_{r}\left[\mathrm{~kg} / \mathrm{m}^{3}\right]$ & 286.9 \\
Density & $m_{r}[\mathrm{~kg}]$ & 1.226 \\
Mass & $t[\mathrm{~m}]$ & 18.39 \\
Heat from metal & $m_{m}[\mathrm{~kg}]$ & \\
Thin profile thickness & $h\left[\mathrm{~W} / \mathrm{m}^{2} / \mathrm{K}\right]$ & 0.0015 \\
Mass & $\rho_{m}\left[\mathrm{~kg} / \mathrm{m}^{3}\right]$ & 18 \\
Heat transfer coeff. & $c_{m}[\mathrm{~J} / \mathrm{kg} / \mathrm{K}]$ & 50 \\
Density & $A_{m}\left[\mathrm{~m}^{2}\right]$ & 2700 \\
Specific heat & & 897 \\
Exposed area equivalent & $c 0[\mathrm{~L} / \mathrm{s}]$ & 4.44 \\
Leakage & $c 1\left[\mathrm{~L} / \mathrm{s} / \mathrm{mbar}^{2}\right.$ & \\
0th order leak. curve coeff. & $c 2[\mathrm{~L} / \mathrm{s} / \mathrm{mbar}]$ & 0 \\
1st order leak. curve coeff. & $A\left[\mathrm{~m}^{2}\right]$ & 0.38 \\
2nd order leak. curve coeff. & & 0.19 \\
Inflow equiv. nozzle area & 0.0002 \\
\hline
\end{tabular}

The dashed lines suffixed "old" in the figures' legend show the behaviour of the former leakage model. Due to linear response of the flow rate expression, the pressure in the room never stops to grow. The new model shows the room is reaching close to equilibrium state, where at certain overpressure (near $20 \mathrm{mbarG}$ ) the volume addition of the agent is almost completely compensated by the leaks of the gas mixture out of the room.

These values of the new model seem to be very close to reality, as the experimental test carried out with the halocarbon agent designated for the application reach very similar values of maximum overpressure. In four consecutive tests, the overpressure reached 18.1, 19.3, 18.4 and 18.5 mbarG.

The temperature of room content $\left(T_{r}\right.$ mix $)$ drops linearly after passing the initial steeper slope. The metal temperature $\left(T_{m}\right)$ follows a similar trend, but as the heat transfer rate from the metal parts is limited, the temperature drops slower. Consequently, this creates a relatively high final temperature difference between metal and gas in the room for both cases, which can cause heat- up of the gas after the agent release period resulting in further rise of pressure.

The temperature inside agent pressure vessel $\left(T_{e}\right)$ shows the effect of liquid only release. Despite the pressure curve, the concave shape is significant. The R403B case shows the tables might be too coarse to have step-free smooth curve, but this is believed to have negligible effects on the important results.

Considering the pressure difference between triple point and critical point for both presented substances (and many others), the change of pressure in the room over time is very low. Therefore, the agent discharge temperature $\left(T_{d}\right)$, being the saturation temperature for a given room pressure, is close to constant. Here the demand for tabulated values density or interpolation algorithm for getting a smooth curve is visible too, but again without a significant effect on the results.

The volumetric concentration estimate reaches far above the needs of the needed levels for reliable function of the system. Environmental analysis results with different inputs may vary. 


\section{Conclusions}

A set of coherent calculations was built for the purpose of designing an integrated fire extinguishing system and analysis of various operating conditions. The overpressure in the extinguished room is checked throughout the timedependent calculation.

There are a few key parts of the work.

At first, a strong constraint of constant mass flow rate is set and carefully proven with experimental results and external References.

The second is the thorough decomposition of the thermodynamic processes and the decisions on how to model them. A summary is given in Figure 3. Many issues have been overcome on the side of using REFPROP tables imported as raw real gas data into MS Excel.

Finally, significant influences were identified. The heat of metal bodies and control volume leakage (Sects. 3.4 and 3.5) are incorporated into the calculation. Two models of leakage were used in the course of development of the tool. The second, more accurate model based on experimental results gives new insight on the process. Since the leakage rate can now reach equilibrium with continual agent addition to the room, the maximum overpressure seems not to ever reach critical values for this particular design.

Results of a case study have been discussed. See Section 4.

The gases may also lose a significant amount of internal energy due to the work being done on the wall's deformation. Analysis of this phenomenon may lead to future enhancements of the design tool.

\section{Nomenclature}

$c \quad$ Specific heat (isobaric) $[\mathrm{J} / \mathrm{kg} / \mathrm{K}]$

$h \quad$ Specific enthalpy $[\mathrm{J} / \mathrm{kg}]$

$m$ Mass [kg]

$p$ Absolute pressure [Pa]

$Q \quad$ Total heat $[\mathrm{J}]$

$q$ Heat per kilogram mass $[\mathrm{J} / \mathrm{kg}]$

$r \quad$ Specific gas constant $[\mathrm{J} / \mathrm{kg} / \mathrm{K}]$

$s \quad$ Specific entropy $[\mathrm{J} / \mathrm{kg} / \mathrm{K}]$

$T$ Absolute temperature $[\mathrm{K}]$ $t$ Time [s]

$V$ Volume $\left[\mathrm{m}^{3}\right]$

$v \quad$ Specific volume $\left[\mathrm{kg} / \mathrm{m}^{3}\right]$

$w$ Flow velocity $[\mathrm{m} / \mathrm{s}]$

$x$ Two-phase quality [-]

$\alpha$ Heat transfer coefficient $\left[\mathrm{W} / \mathrm{m}^{2} / \mathrm{K}\right]$

Indices:

d Agent discharge state

$e \quad$ Extinguishing agent state

init Initial

$l \quad$ Leak

liq Liquid

$m$ Metal

$n \quad$ Time step number

vap Vapour

$r \quad$ Room

sat Saturation state

This work was supported by the project of specific research UWB SGS-2019-021. Authors would like to express their special thanks to the company Safran Cabin CZ s.r.o. as well as to professor František Maršík from IT CAS.

\section{References}

[1] L. Hurda, R. Matas, Liquefied extinguishing agent discharge to an overpressure-sensitive enclosed volume, MATEC Web Conf. 168, 07010 (2018)

[2] A.G. Linde, Retrofit Guide, available at https://www.lindegas.com/internet.global.lindegas.glo-bal/en/images/Refriger ant\%20Retrofit\%20guides17_109809.pdf?v=5.0, 2017

[3] M. Ahmad, L. Buit, O. Florisson, C. Hulsbosch-Dam, M. Bögemann-van Osch, M. Spruijt, F. Davolio, Experimental Investigation of CO2 Outflow from a High-pressure Reservoir, Energy Procedia 37, 3005-3017 (2013)

[4] National Fire Protection Association, NFPA Code 12A: Standard on Halon 1301 fire extinguishing systems, 2018

[5] R.M. Stephenson, S. Malanowski, Handbook of the Thermodynamics of Organic Compounds, Elsevier, New York, 1987

Cite this article as: L. Hurda, R. Matas, M. Redmer, Analysis of room overpressure after launching an integrated extinguishing system, Mechanics \& Industry 20, 706 (2019) 